\title{
KINKS IN ONE-DIMENSIONAL TWO-SUBLATTICE ANTIFERROMAGNET WITH BIQUADRATIC EXCHANGE INTERACTION
}

\author{
I.V. BARYAKHTAR \\ B.I. Verkin Institute for Temperature Physics and Engineering \\ 47 Lenin Avenue, Kharkov, 310164, Ukraine
}

\begin{abstract}
Nonlinear dynamics of an antiferromagnet in continuum approximation on the basis of the Heisenberg Hamilionian for two sublattices with biquadratic exchange interaction in each sublattice and single ion anisotropy is considered. Exact particular solution, describing the stationary kink movement, including the region $v \geq c$, is found.
\end{abstract}

PACS numbers: 75.40.Gb, 75.50.Ee

\section{Introduction}

Nonlinear wave phenomena of an antiferromagnet have been actively investigated in the last years (see [1-3]). Three possibilities are known to analyse nonlinear waves in such magnetics: (i) on the basis of the system of the Landau-Lifshitz equations [4, 5]; (ii) on the basis of the Heisenberg Hamiltonian [6]; (iii) on the basis of analysis of an order parameter of antiferromagnet [7]. In the simplest case these models lead to the sine-Gordon equation. The solutions of sine-Gordon equation describe such experimental results as a domain wall movement in weak ferromagnet i.e. ferromagnet with Dzyaloshinsky interaction [3] or soliton excitations in quasi-one-dimensional chains like TMMC [6]. However, the analysis of soliton dynamics with velocities $v$ about the minimum phase velocity of spin wave $c$ is impossible because soliton width tends to zero when $v \rightarrow c$. Therefore, it is necessary to take into account high order derivatives on space coordinates to finite soliton width in this region of parameter $v$. In other words, it is necessary to include in our consideration the biquadratic exchange interaction in an antiferromagnet Hamiltonian. The problem of simplification of the system of the Landau-Lifshitz equations for antiferromagnet if high order derivatives are taken into account was considered in [8].

On the other hand, a biquadratic exchange interaction is significant for high spin systems $[9,10]$. The role of biquadratic interaction for nonlinear dynamics of ferromagnets was investigated in [11-14]. 
In this paper we will consider nonlinear dynamic of an antiferromagnet in a continuum approximation on the basis of the Heisenberg Hamiltonian for two sublattices with biquadratic exchange interaction in each sublattices and single-ion anisotropy.

\section{Model}

Let us consider the following Hamiltonian:

$$
\begin{aligned}
\mathcal{H}= & \sum_{n}\left\{-J_{0}\left(S_{1, n} \cdot S_{1, n+1}+S_{2, n} \cdot S_{2, n+1}\right)\right. \\
& +J_{1}\left(S_{1, n+1} \cdot S_{2, n-1}+S_{2, n+1} \cdot S_{1, n-1}\right) \\
& \left.+J_{2}\left[\left(S_{1, n+1} \cdot S_{1, n-1}\right)^{2}+\left(S_{2, n} \cdot S_{2, n-1}\right)^{2}\right]-K\left[\left(S_{1, n}^{z}\right)^{2}+\left(S_{2, n}^{z}\right)^{2}\right]\right\}
\end{aligned}
$$

where $J_{0}, J_{1}, K>0$.

The equation of motion can be written as

$$
\begin{aligned}
\frac{\partial S_{1, m}}{\partial t} & =g\left\{S_{1, m}, \mathcal{H}\right\}, \\
\frac{\partial S_{2, m}}{\partial t} & =g\left\{S_{2, m}, \mathcal{H}\right\},
\end{aligned}
$$

where the Poisson brackets are determined in a standard way

$$
\{A, B\}=\varepsilon_{\nu, \mu, \xi} \sum_{n} \frac{\mathrm{d} A}{\mathrm{~d} S_{n}^{\nu}} \frac{\mathrm{d} B}{\mathrm{~d} S_{n}^{\mu}} S_{n}^{\xi},
$$

where $\nu, \mu, \xi=x, y, z$.

Substituting Hamiltonian (1) in Eqs. (2), (3) we obtain the following equations:

$$
\begin{aligned}
& \frac{\partial S_{1, m}}{\partial t}=-g\left[S _ { 1 , m } \left\{J_{0}\left(S_{1, m+1}+S_{1, m-1}\right)-J_{1}\left(S_{2, m+1}+S_{2, m-1}\right)\right.\right. \\
& \left.\left.\quad+J_{2}\left(S_{1, m-1}\left(S_{1, m-1} \cdot S_{1, m}\right)+S_{1, m+1}\left(S_{1, m+1} \cdot S_{2, m}\right)\right)\right\}+K S_{1, m}^{z} e_{z}\right], \\
& \frac{\partial S_{2, m}}{\partial t}=-g\left[S _ { 2 , m } \left\{J_{0}\left(S_{2, m+1}+S_{2, m-1}\right)-J_{1}\left(S_{1, m+1}+S_{1, m-1}\right)\right.\right. \\
& \left.\quad+J_{2}\left(S_{2, m-1}\left(S_{2, m-1} \cdot S_{2, m}+S_{2, m+1}\left(S_{2, m+1} \cdot S_{2, m}\right)\right)+K S_{2, m}^{z} e_{z}\right\}\right],
\end{aligned}
$$

Let us introduce vectors $\boldsymbol{S}_{m}$ and $\boldsymbol{L}_{m}$ as

$$
\boldsymbol{S}_{m}=\frac{\boldsymbol{S}_{1, m}+\boldsymbol{S}_{2, m}}{2 S_{0}}, \quad \boldsymbol{L}_{m}=\frac{\boldsymbol{S}_{1, m}-S_{2, m}}{2 S_{0}},
$$

where $S_{1, n}^{2}=S_{2, n}^{2}=S_{0}^{2}$.

Then Eqs. (5), (6) can be simplified in a continuum description if we use approximation usual for antiferromagnet

$$
|S| \ll|L|, \quad L^{2}=1
$$

The following formula can be obtained from the sum of Eqs. (5), (6):

$$
\boldsymbol{S}=\frac{1}{g J_{0} S_{0}} \frac{\partial \boldsymbol{L}}{\partial t} \times \boldsymbol{L}
$$


The difference of Eqs. (5), (6) can be reduced to the following equation for vector $\boldsymbol{L}$ :

$$
\begin{gathered}
\frac{\alpha}{c^{2}}\left[L \times \frac{\partial^{2} L}{\partial t^{2}}\right]-\alpha\left[L \times \frac{\partial^{2} L}{\partial x^{2}}\right]+\sigma_{1}\left[L \times \frac{\partial^{4} L}{\partial x^{4}}\right]-\sigma_{2}\left[L \times\left(\frac{\partial L}{\partial t}\right)^{2} \frac{\partial^{2} L}{\partial x^{2}}\right] \\
-\sigma_{3}\left[L \times \frac{\partial L}{\partial x}\left(\frac{\partial^{3} L}{\partial x^{3}}\right)\right]-K\left[L \times L_{z} e_{z}\right]=0,
\end{gathered}
$$

here

$$
\begin{aligned}
& \alpha=\left(J_{0}-J_{2} S_{0}^{2}-J_{1}\right) a^{2}, \quad \sigma_{1}=\left(J_{0}-J_{1}-J_{2} S_{0}^{2}\right) a^{4} / 12, \\
& \sigma_{2}=J_{2} a^{4} / 2, \quad \sigma_{3}=J_{2} a^{4} / 3,
\end{aligned}
$$

where $a$ is the lattice constant.

Equation (8) is the generalization of anisotropic $\sigma$ model of $n$-field.

\section{Solution}

Let us analyse Eq. (9) in angular variables for vector $L\left(L_{x}=\sin \theta \cos \phi\right.$, $\left.L_{y}=\sin \theta \sin \phi, L_{z}=\cos \theta\right)$. It is easy to see that if

$$
\phi=0 \text { or } \frac{\pi}{2} \text {, }
$$

the equation for $\theta$ is

$$
-\frac{\alpha}{c^{2}} \frac{\partial^{2} \theta}{\partial t^{2}}+\alpha \frac{\partial^{2} \theta}{\partial x^{2}}+\sigma_{1} \frac{\partial^{4} \theta}{\partial x^{4}}-\gamma \frac{\partial^{2} \theta}{\partial x^{2}}\left(\frac{\partial \theta}{\partial x}\right)^{2}-K \sin \theta \cos \theta=0
$$

where

$$
\gamma=6 \sigma_{1}+\sigma_{2}+3 \sigma_{3} .
$$

Equation (11) is the higher order sine-Gordon equation and was obtained as a generalization of the Frenkel-Kondorova model. For mathematical aspects of this equation see for example in [15]. If $\gamma=-6 \sigma_{1}$ i.e.

$$
J_{0}=J_{1}+\frac{5 J_{2}}{2}
$$

it is possible to find the exact stationary wave type solution of Eq. (11). This solution can be written in the form [16]:

$$
\tan \frac{\theta}{2}=\exp \left(-\frac{\xi}{l_{0}}\right)
$$

where $\xi=x-v t$, and

$$
l_{0}^{2}=\frac{x_{0}^{2}}{2}\left\{\left(1-\frac{v^{2}}{c^{2}}\right)+\left[\left(1-\frac{v^{2}}{c^{2}}\right)^{2}+\frac{4 \sigma_{1} K}{\alpha^{2}}\right]^{\frac{1}{2}}\right\} .
$$

Here $l_{0}$ is the kink width, $x_{0}=(\alpha / K)^{1 / 2}$ is characteristic magnetic length i.e. the kink width when $v=0$ and $\sigma_{1}=\gamma=0$. In the case considered the formulas (12), (13) describe the kink moving with velocities from 0 to $\infty$, i.e. both larger and smaller than $c$. 
The solution (12), (13) can be used for analysis of kink movement in an antiferromagnet with generalized energy of anisotropy and at $\sigma_{1} \neq-6 \gamma$ if we consider these solution' as a trial function. Really, if we choose Hamiltonian describing anisotropy as

$$
\mathcal{H}_{1}=-\sum_{n}\left\{K_{1}\left(S_{1, n}^{z}\right)^{2}+K_{1}\left(S_{2, n}^{z}\right)^{2}+K_{2}\left(S_{1, n}^{z}\right)^{4}+K_{2}\left(S_{2, n}^{z}\right)^{4}\right\} .
$$

Lagrangian density function, corresponding to Eq. (11), has the form

$$
\begin{aligned}
\mathcal{L}= & \frac{1}{2}\left\{\frac{\alpha}{c^{2}}\left(\frac{\partial \theta}{\partial t}\right)^{2}-\alpha\left(\frac{\partial \theta}{\partial x}\right)^{2}+\sigma_{1}\left(\frac{\partial^{2} \theta}{\partial x^{2}}\right)^{2}+\frac{\gamma}{6}\left(\frac{\partial \theta}{\partial x}\right)^{4}\right. \\
& \left.-K_{1} \sin ^{2} \theta-K_{2} \sin ^{4} \theta\right\}
\end{aligned}
$$

Then from the condition of extremes of Lagrangian density function (15), we can obtain the following expression for kink width:

$$
\begin{aligned}
& l_{0}^{2}=\frac{x_{0}^{2}}{2\left(1+2 K_{2} / 3 K_{2}\right)} \\
& \times\left\{\left(1-\frac{v^{2}}{c^{2}}\right)+\left[\left(1-\frac{v^{2}}{c^{2}}\right)^{2}+\frac{4}{3 \alpha^{2}}\left(\gamma+9 \sigma_{1}\right)\left(K_{1}+\frac{2}{3} K_{2}\right)\right]^{\frac{1}{2}}\right\} .
\end{aligned}
$$

In order to estimate the kink width when $v \approx c$ we can use formula (16). It is easy to see that if $K_{2}=0, \gamma=-6 \sigma_{1}$, formula (16) will turn into formula (13).

\section{Summary and conclusions}

In this paper we have discussed the role of the biquadratic exchange interaction in the nonlinear dynamics of the continuum longitudinal anisotropic two sublattices Heisenberg antiferromagnet chain. We conclude that the equation of motion with this interaction gives generalization of the phenomenological equation of the antiferromagnet nonlinear dynamics - anisotropic $\sigma$ model of $n$-field. Its exact particular solution describes the stationary kink movement including the region $v \geq c$.

Stationary kink movement will be realized when the action of the external magnetic field is compensated by the domain boundary bracing force. A peculiarity of the stationary kink movement with $v \geq c$ is the Cherenkov radiation of spin waves [1]. Consequently, the kink braking force and corresponding magnetic field increase. This fact leads to additional difficulties in the experimental investigation of the effect considered. Nevertheless, in our opinion the solution (12), (13) shows the possibility of real superlimit kink movement in an antiferromagnet. In any case this solution describes the kink movement in the region $v \approx c$ well investigated experimentally, since the kink width remains finite when $v \rightarrow c$ and not tends to zero in contrast to usual sine-Gordon model. 


\section{Acknowledgments}

It is a pleasure for the author to thank B. Baumgartner and H. Grosse for their interest and helpful discussions and D.V. Meshcheryakov for discussions. He also would like to thank Institute for Theoretical Physics of Vienna University for hospitality. This work has been supported by the Federal Ministry of Science and Research of Republic of Austria and partially by Ukrainian doctor's society in Austria.

\section{References}

[1] V.G. Baryakhtar, B.A. Ivanov, M.V. Chetkin, Usp. Fiz. Nauk 146, 417 (1985).

[2] A.M. Mikhailov, in: Solitons, Eds. S.E. Trullinger, V.E. Zakharov, V.L. Pokrovsky, Elsevier, New York 1986.

[3] A.M. Kosevich, B.A. Ivanov, A.S. Kovalev, Phys. Rep. 194, 119 (1990).

[4] I.V. Baryakhtar, B.A. Ivanov, Fiz. Nizk. Temp. 5, 759 (1979).

[5] I.V. Baryakhtar, B.A. Ivanov, Phys. Lett. A 98, 222 (1983).

[6] H.J. Mikeshka, J. Phys. C 13, 2913 (1980).

[7] A.F. Andreev, V.I. Marchenko, Usp. Fiz. Nauk 130, 1509 (1980).

[8] I.V. Baryakhtar, Phys. Lett. A 168, 331 (1992).

[9] P.W. Anderson, Phys. Rev. 115, 2 (1959).

[10] E.L. Nagaev, Magnets with Complicated Exchange Interactions, Nauka, Moskva 1988 [in Russian].

[11] P. Ferrer, Physica B 132, 56 (1985).

[12] V.S. Ostrovskii, Zh. Eksp. Teor. Fiz. 91, 1690 (1986).

[13] A.E. Borovik, V. Popkov, Zh. Eksp. Teor. Fiz. 71, 177 (1990).

[14] A.I. Gaponov, B.A. Ivanov, A.N. Kichizhev, Yu.N. Mitsai, Fiz. Nizk. Temp. 17, 84 (1991).

[15] P. Golod, Fiz. Mnogochastichnyh System 7, 30 (1985).

[16] A.M. Kosevich, A.S. Kovalev, Solid State Commun. 12, 763 (1973). 